\title{
Asymptotic Behaviour of Spin-Momentum Distribution Observables for Fermion Fields with Cut-Off Self-Coupling*
}

\author{
E. PrugovečKI \\ Department of Mathematics, University of Toronto, Canada \\ E. B. MaNOUKian \\ Department of Physics, University of Toronto, Canada \\ Received September 6; in revised form October 7, 1971
}

\begin{abstract}
In a previous paper asymptotic creation and annhilation operators $a_{ \pm}^{\sharp}$ have been constructed by the Kato-Mugibayashi method from the creation and annihilation operators $a^{\#}$ for spin $\frac{1}{2}$ fields with an interaction Hamiltonian density which is an evendegree polynomial in the field with ultra-violet cut-off and its derivatives. For any eigenvector $\Phi$ of the total Hamiltonian $H=H_{0}+H_{I}$ partial isometries $\Omega_{ \pm}$have been defined so that $a_{ \pm}^{\#}$ equal $\Omega_{ \pm} a^{\sharp} \Omega_{ \pm}^{*}$ on the ranges $\mathscr{F}_{ \pm}$of $\Omega_{ \pm}$. Since the existence of a groundstate of $H$ has been proved, the existence of at least one pair $\Omega_{ \pm}$follows. The purpose of this paper is to show that for any $\Psi \in \mathscr{F}_{ \pm}$orthogonal to $\Phi$ the distribution of spins and momenta of the interacting Schrödinger states $\exp [-i t H] \Omega_{ \pm} \Psi$ approaches for $t \rightarrow \mp \infty$ the distributions of spins and momenta of the free state $\exp \left[-i t H_{0}\right] \Psi$ if a wave-amplitude renormalization is carried out in $\mathscr{F}_{ \pm}$. This is achieved by studying the expectation values of the operators in the maximally abelian $W^{*}$-algebra $\mathfrak{A}(\tilde{\mathcal{O}})$ generated by operators of the form $\int \varrho a^{*} a$, in terms of which any information about spins and momenta can be expressed.
\end{abstract}

\section{Introduction}

The aim of this paper is to demonstrate that the concept of physical wave-operators introduced in Ref. [1] in order to deal with the scattering problem for long-range potentials [2] can be applied also to constructive quantum field theory. This presents the hope for a unified quantum scattering theory formulated in terms of such operators, which would apply to the relativistic as well as non-relativistic case.

The class of models under study [3] are the spin $\frac{1}{2}$ fields $\psi(x)$ which self-interact via an interaction Hamiltonian

$$
H_{I, \kappa, v}=\int_{\mathbb{R}^{3}} v(\boldsymbol{x}) H_{I, \kappa}(\boldsymbol{x}) d^{3} \boldsymbol{x}
$$

\footnotetext{
* Supported in part by the National Research Council of Canada.
} 
with a finite ultraviolet cut-off $\kappa$ and a space cut-off $v(x)$ of compact support. Dealing with one field rather than with the more general and more realistic case of $n$ interacting spin $\frac{1}{2}$ fields simplifies the notation without presenting a serious limitation on the techniques and results, which stay valid in the general case.

Let us adopt a very general approach to scattering theory by assuming that the interacting system is described in a Hilbert space $\mathscr{H}$, while the asymptotic cases in which there is no interaction between all parts of the system are described in the Hilbert spaces $\mathscr{H}^{\text {ex }}$, ex $=$ in, out, which are not necessarily identical with $\mathscr{H}$. The family of observables in $\mathscr{H}$ and $\mathscr{H}^{\text {ex }}$ will be denoted by $\mathcal{O}$ and $\mathcal{O}^{\text {ex }}$, respectively. It is straightforward to generalize the procedure for defining physical wave operators in Ref. [1] by assuming first the existence of a mapping $A \rightarrow A^{\text {ex }}$ of $\mathcal{O}$ onto $\mathcal{O}^{\text {ex }}$ (determined on physical grounds [1]) and then stating that any pair $\Omega_{ \pm}$of linear one-to-one mappings of $\mathscr{H}^{\text {in }}$ onto closed subspaces $\underline{R}_{ \pm}$ of $\mathscr{H}$ represents physical wave operators if $\Omega_{-}^{*} \Omega_{+}$is a partial isometry from $\mathscr{H}^{\text {in }}$ to $\mathscr{H}^{\text {out }}$ and if

$$
\lim _{t \rightarrow \mp \infty}\left|\left\langle e^{-i t H} \Omega_{ \pm} \Psi \mid A e^{-i t H} \Omega_{ \pm} \Psi\right\rangle-\left\langle e^{-i t H_{0}} \Psi \mid A e^{-i t H_{0}}\right\rangle_{\mathrm{ex}}\right|=0
$$

for any bounded operator $A \in \mathcal{O}$ and state-vectors $\Omega_{ \pm} \Psi \in \underline{R}_{ \pm}$.

In the case treated in this paper, $\mathscr{H}^{\text {in }}=\mathscr{H}^{\text {out }}$ are chosen to be identical to the Fock space $\mathscr{F}$ (Section 2), while $\mathscr{H}$ consists of a linear subspace of $\mathscr{F}$ supplied with a "renormalized" inner-product $\langle. \mid .\rangle_{\text {ren }}$ which is equal to the original inner product multiplied by $e^{\Lambda}$, where $e^{\Lambda / 2}$ is a waveamplitude renormalization constant. The set $\mathcal{O}$ of observables considered in this paper is $\mathcal{O}=\left\{H_{\kappa, v}\right\} \cup \mathcal{O}_{0}$, and corresponding $\mathcal{O}^{\mathrm{ex}}=\left\{H_{0}\right\} \cup \mathcal{O}_{0}$, where $\mathcal{O}_{0}$ is the sub-algebra of all self-adjoint elements in the $W^{*}$-algebra $\mathfrak{U}(\tilde{\mathcal{O}})$ generated by the spin-momentum distribution observables [3]. The choice of $\mathfrak{U}(\tilde{\mathcal{O}})$ instead of the algebra of local observables is deliberate, since it presents us with a maximally abelian algebra [3] of observables in terms of which any measurement of spins and momenta of any finite number of particles can be described. Since spin and momentum are ideally suited to scattering experiments, and since $S$-matrix computations are always carried out in the momentum rather than configuration space, this choice seems most appropriate.

The wave operators of the models considered are partial isometries on $\mathscr{F}$, and are defined by means of asymptotic creation and annhilation operators [4-6, 3] and of eigenvectors of $H_{\kappa, v}$. Since we have no information about the point spectrum of $H_{\kappa, v}$ beyond the existence of the vacuum energy $[3,7]$ as the lowest eigenvalue, we cannot establish uniqueness of $\Omega_{ \pm}$. In general, to each eigenvalue of $H_{\kappa, v}$ would correspond a different pair of physical wave operators. 
In Section 3 we establish the main result of the paper (Theorem 3.1) which implies that if $\Omega_{ \pm} \Psi$ are orthogonal to the ground state of $H_{\kappa, v}$ then

$$
\lim _{t \rightarrow \mp \infty}\left\langle\Omega_{ \pm} \Psi \mid e^{i t H_{\kappa, v}} A e^{-i t H_{\kappa, v}} \Omega_{ \pm} \Psi\right\rangle_{\text {ren }}=\langle\Psi \mid A \Psi\rangle
$$

for any $A \in \mathfrak{Q}(\tilde{\mathcal{O}})$ corresponding to a finite number of particles. In view of (1.2) and the commutativity of $H_{0}$ and $\mathfrak{A}(\mathcal{O})$, this means that $\Omega_{ \pm}$are physical wave operators, and therefore $[1,8]$ that the interacting states $\exp \left[-i t H_{\kappa, v}\right] \Omega_{ \pm} \Psi$ describe asymptotically in time for $t \rightarrow \mp \infty$ respectively the same distribution of spins and momenta as the free states $\exp \left[-i t H_{0}\right] \Psi$.

In the conclusion (Section 4) we discuss some further physical implications of the derived results.

\section{Fock Space and Summary of Previous Results}

To simplify the notation we introduce the measures $\mu$ and $\mu_{0}$ on the Borel sets in $\mathbb{R}^{4}$ and $\mathbb{R}^{5}$, respectively:

$$
\begin{gathered}
d \mu(\boldsymbol{k}, \sigma)=\frac{d^{3} \boldsymbol{k}}{\omega(\boldsymbol{k})} d \mu_{1}(\sigma), \quad \omega(\boldsymbol{k})=\sqrt{\boldsymbol{k}^{2}+m_{0}^{2}}, \\
d \mu_{0}(\boldsymbol{k}, \sigma, v)=d \mu(\boldsymbol{k}, \sigma) d \mu_{2}(v) .
\end{gathered}
$$

Here $m_{0}$ is the mass of the Fermion, while $\mu_{1}$ and $\mu_{2}$ are measures in $\mathbb{R}^{1}$ with supports on the sets $\left\{-\frac{1}{2},+\frac{1}{2}\right\}$ and $\{-1,+1\}$, respectively, and such that

$$
\mu_{1}\left(\left\{ \pm \frac{1}{2}\right\}\right)=\mu_{2}(\{ \pm 1\})=1
$$

where $\sigma$ and $v$ denote spin and particle-antiparticle indices, respectively.

The single particle space $\mathscr{F}_{1,0}$ and the single antiparticle space $\mathscr{F}_{0,1}$ in the momentum representation are both taken to be identical to $L_{\mu}^{2}\left(\mathbb{R}^{4}\right)$. The space $\mathscr{F}_{m, n}$ of $m$ Fermions and $n$ antifermions, $m+n>0$, is the space

$$
\mathscr{F}_{m, n}=\left[\mathscr{F}_{1,0} \otimes_{A^{m}}\right] \otimes\left[\mathscr{F}_{0,1} \otimes_{A^{n}}\right]
$$

where $\otimes_{A}$ denotes the antisymmetric tensor product, and for $m=0$ and $n=0$ we identify the corresponding tensor product with the one dimensional space $\mathscr{F}_{0,0}$ generated by the Fock vacuum $\Phi_{0,0}$. The Fock space is

$$
\mathscr{F}=\bigoplus_{m, n=0}^{\infty} \mathscr{F}_{m, n} .
$$

On proper subspaces of $\mathscr{F}$ [3], the creators and annhilators as sesquilinear forms satisfy:

$$
\left[a_{v}(\boldsymbol{k}, \sigma), a_{v}^{*},\left(\boldsymbol{k}^{\prime}, \sigma^{\prime}\right)\right]_{+}=\delta_{v v}, \delta_{\sigma \sigma^{\prime}} \omega(\boldsymbol{k}) \delta\left(\boldsymbol{k}-\boldsymbol{k}^{\prime}\right)
$$

with all the other anticommutators being zero.

10 Commun. math. Phys., Vol. 24 
If $U_{t}=\exp \left[i t H_{\kappa, v}\right] \exp \left[-i t H_{0}\right]$ then for any $f(\boldsymbol{k}, \sigma) \in L_{\mu}^{2}\left(\mathbb{R}^{4}\right)$

$$
a_{v \pm}^{\#}(f)=u-\lim _{t \rightarrow \mp \infty} U_{t} a_{v}^{\#}(f) U_{t}^{*}
$$

exist $[3,4-6]$, and satisfy the same anticommutation relations given in (2.4). Let $\Phi$ be any eigenvector of $H_{\kappa, v}$ and denote by $\mathscr{F}_{ \pm}(\Phi)$ the closed linear subspace of $\mathscr{F}$ spanned by the vectors

$$
a_{v_{ \pm} \pm}^{*}\left(f_{1}\right) \ldots a_{v_{n \pm}}^{*}\left(f_{n}\right) \Phi
$$

corresponding to all $f_{1}, \ldots, f_{n} \in L_{\mu}^{2}\left(\mathbb{R}^{4}\right)$ and $v_{1}, \ldots, v_{n} \in\{-1,+1\}$ and for all $n=0,1,2, \ldots$.

When $H_{\kappa, v}$ is restricted to $\mathscr{F}_{ \pm}(\Phi)$ it is given by [3]

$$
H_{\kappa, v}=\eta \mathbf{1}+\int_{\mathbb{R}^{5}} \omega(\boldsymbol{k}) a_{v_{ \pm}}^{*}(\boldsymbol{k}, \sigma) a_{v_{ \pm}}(\boldsymbol{k}, \sigma) d \mu_{0}(\boldsymbol{k}, \sigma, v)
$$

where $\eta$ is the eigenvalue of $H_{\kappa, v}$ corresponding to $\Phi$.

The wave operators $\Omega_{ \pm}(\Phi)$ are defined $[3,6]$ by the relation

$$
\Omega_{ \pm}(\Phi) a_{v_{1}}^{*}\left(f_{1}\right) \ldots a_{v_{n}}^{*}\left(f_{n}\right) \Phi_{0,0}=a_{v_{1 \pm}}^{*}\left(f_{1}\right) \ldots a_{v_{n \pm}}^{*}\left(f_{n}\right) \Phi .
$$

Let $E_{ \pm}(\Phi)$ be the projection of $\mathscr{F}$ onto $\mathscr{F}_{ \pm}(\Phi)$. Then $\Omega_{ \pm}(\Phi)$ satisfy $[3,6]$ the following relations:

$$
\begin{aligned}
\Omega_{ \pm}^{*}(\Phi) \Omega_{ \pm}(\Phi) & =\mathbf{1} \\
\Omega_{ \pm}(\Phi) \Omega_{ \pm}^{*}(\Phi) & =E_{ \pm}(\Phi), \\
\exp \left[i t\left(H_{\kappa, v}-\eta\right)\right] \Omega_{ \pm}(\Phi) & =\Omega_{ \pm}(\Phi) \exp \left[i t H_{0}\right], \quad t \in \mathbb{R}^{1} .
\end{aligned}
$$

From (2.5) it also follows [3] that

$$
\begin{gathered}
u-\lim _{t \rightarrow \mp \infty} U_{t} a_{v_{1}}^{\#}\left(f_{1}\right) \ldots a_{v_{l}}^{\#}\left(f_{l}\right) U_{t}^{*} E_{ \pm}(\Phi) \\
=\Omega_{ \pm}(\Phi) a_{v_{1}}^{\#}\left(f_{1}\right) \ldots a_{v_{l}}^{\#}\left(f_{l}\right) \Omega_{ \pm}^{*}(\Phi)
\end{gathered}
$$

for all $f_{1}, \ldots, f_{l} \in L_{\mu}^{2}\left(\mathbb{R}^{4}\right), \quad l=1,2, \ldots$.

Let us introduce the operators

$$
P_{\varrho}=\int_{\mathbb{R}^{5}} \varrho(\boldsymbol{k}, \sigma, v) a_{v}^{*}(\boldsymbol{k}, \sigma) a_{v}(\boldsymbol{k}, \sigma) d \mu_{0}(\boldsymbol{k}, \sigma, v)
$$

and define the family of spin-momentum distribution observables $\tilde{\mathcal{O}}$ to be the set [3] of all self-adjoint operators

$$
A=E_{m, n} P_{\varrho}
$$

for all finite $m$ and $n$ and all real functions $\varrho$ which are essentially bounded with respect to $\mu_{0}$; here $E_{m, n}$ denotes the projection of $\mathscr{F}$ onto $\mathscr{F}_{m, n}$. Then the following theorem has been proved [3]. 
Theorem 2.1. The $W^{*}$-algebra $\mathfrak{A}(\tilde{\mathcal{O}})$ generated by $\tilde{\mathcal{O}}$ is maximally abelian and any $A \in \mathfrak{A}(\tilde{\mathcal{O}})$ when restricted to $\mathscr{F}_{m, n}$ is given by

$$
A \Psi_{m, n}=\alpha_{m, n}\left(\boldsymbol{k}_{1}, \sigma_{1}, \ldots, \boldsymbol{k}_{m+n}, \sigma_{m+n}\right) \Psi_{m, n},
$$

where $\alpha_{m, n}$ is a Borel measurable function on $\mathbb{R}^{4(m+n)}$ and is symmetric under permutations of the first $m$ and of the last $n$ four-variables. The function $\alpha_{m, n}$ is also essentially bounded with respect to the product measure $\mu^{\times(m+n)}$.

Since by definition $A$ corresponds to a finite number of particles,

$$
\|A\|=\max _{m, n=0,1, \ldots}\left\{\underset{\mathbb{R}^{4}(m+n)}{\operatorname{ess}-\sup }\left|\alpha_{m, n}\left(\boldsymbol{k}_{1}, \sigma_{1}, \ldots, \boldsymbol{k}_{m+n}, \sigma_{m+n}\right)\right|\right\}
$$

exist [3]. The *-algebra generated by $\tilde{\mathcal{O}}$ will be denoted by $\mathfrak{A}_{0}(\tilde{\mathcal{O}})$.

Note that Theorem 2.1 states that the spin-momentum distribution observables $\tilde{\mathcal{O}}$ form a complete set of observables.

The interaction density $H_{I, \kappa}(x)$ is chosen to be [3] a polynomial

$$
T(\bar{\psi}, \psi)=: P\left[\bar{\psi}, \psi, \frac{\partial \bar{\psi}}{\partial x_{r}}, \frac{\partial \psi}{\partial x_{r}}, \ldots, \frac{\partial^{k} \psi}{\partial x_{r_{1}} \ldots \partial x_{r_{k}}}\right]:
$$

consisting of products of bilinear forms in $\bar{\psi}$ or its space derivatives as left variables and $\psi$ or its space derivatives as right variables, all taken at time $t=0 ; \psi$ and $\bar{\psi}$ are the well known configuration representation fields with ultraviolett cut-off $\kappa$.

\section{The Asymptotic Behaviour in Time of the Heisenberg-Picture Spin- Momentum Distribution Observables}

In this section we intend to show that the operators $\Omega_{ \pm}(\Phi)$ are physical wave operators [1] with respect to the family $\tilde{\mathcal{O}}$ of observables, by proving that the Schrödinger free state $e^{-i t H_{0}} \Psi$ is the incoming and outgoing physical asymptotic state (with respect to $\tilde{\mathcal{O}}$ ) of the Schrödinger interacting states

$$
e^{-i t H_{\kappa}, v} \Omega_{ \pm}(\Phi)\left[E_{ \pm}(\Phi)-|\Phi\rangle\langle\Phi|\right]
$$

for $t \rightarrow \mp, \infty$, respectively. As we have mentioned in the introduction, this will be possible only if we introduce in $\mathscr{F}_{ \pm}^{\prime}(\Phi)=\mathscr{F}_{ \pm}(\Phi)-[\Phi]$ (where $[\Phi]$ is the one-dimensional subspace spanned by $\Phi)$ the renormalized inner product

$$
\langle. \mid .\rangle_{\mathrm{ren}}=e^{\Lambda(\Phi)}\langle. \mid .\rangle
$$

which represents a wave-function amplitude renormalization ([9], p. 112) with

$$
\Lambda(\Phi)=-2 \ln \left|\left\langle\Phi \mid \Phi_{0,0}\right\rangle\right|,\|\Phi\|=\left\|\Phi_{0,0}\right\|=1 .
$$


The implicit assumption is that, for the given cut-offs $\kappa$ and $v, \Lambda(\Phi)$ is finite. Then the asymptotic condition, which represents the criterion for $\Omega_{ \pm}(\Phi)$ to be physical wave-operators with respect to $\mathcal{O}$, is a consequence of the following theorem (cf. [1]).

Theorem 3.1. Suppose that $\Phi$ is an eigenvector of $H_{\kappa, v}$, and that $|\Phi\rangle\langle\Phi|$ is the projection onto $\Phi$. Then for any $A \in \mathfrak{A}(\tilde{\mathcal{O}})$ with only a finite number of non-zero components $\left(A \mid \mathscr{F}_{m, n}\right)$ in the subspaces $\mathscr{F}_{m, n}$ the following is true,

$$
\begin{aligned}
& \underset{\substack{w \rightarrow \mp \infty \\
t \rightarrow \lim _{ \pm}}}{ }\left(E_{ \pm}(\Phi)-|\Phi\rangle\langle\Phi|\right) e^{i H_{\kappa, v} t} A e^{-i H_{\kappa, v} t}\left(E_{ \pm}(\Phi)-|\Phi\rangle\langle\Phi|\right) \\
& \quad=e^{-\Lambda(\Phi)}\left(E_{ \pm}(\Phi)-|\Phi\rangle\langle\Phi|\right) \Omega_{ \pm}(\Phi) A \Omega_{ \pm}^{*}(\Phi)\left(E_{ \pm}(\Phi)-|\Phi\rangle\langle\Phi|\right)
\end{aligned}
$$

where $\Lambda(\Phi)$ is defined in (3.2).

Let us denote by $E_{m, n}=\chi_{\{(m, n)\}}\left(N_{+1}, N_{-1}\right)$ the projection onto $\mathscr{F}_{m, n}$. We shall establish first the theorem for observables of the form $E_{m, n} \bar{P}_{\varrho_{1}} \ldots \bar{P}_{\varrho l}$. This will be achieved in a few stages, by proving in the process an auxiliary theorem and three lemmas.

Before proceeding with that proof we note that (3.3) does not hold if $E_{ \pm}(\Phi)$ is retained in place of $E_{ \pm}(\Phi)-|\Phi\rangle\langle\Phi|$ since for $A=E_{m, n} \in \mathfrak{A}_{0}(\tilde{\mathcal{O}})$, $m+n>0$, we have

$$
\begin{aligned}
& \underset{\substack{W-\mp \\
t \rightarrow \infty}}{ }|\Phi\rangle\left\langle\Phi\left|e^{i H_{\kappa, v} t} E_{m, n} e^{-i H_{\kappa, v} t}\right| \Phi\right\rangle\langle\Phi| \\
& \quad=\left\langle\Phi \mid E_{m, n} \Phi\right\rangle|\Phi\rangle\langle\Phi| \\
& \quad \neq e^{-\Lambda(\Phi)}|\Phi\rangle\left\langle\Phi\left|\Omega_{ \pm}(\Phi) E_{m, n} \Omega_{ \pm}^{*}(\Phi)\right| \Phi\right\rangle\langle\Phi| \\
& \quad=e^{-\Lambda(\Phi)}\left\langle\Phi_{0,0} \mid E_{m, n} \Phi_{0,0}\right\rangle|\Phi\rangle\langle\Phi|=0 .
\end{aligned}
$$

Furthermore, because of the in general non-zero renormalization constant $\Lambda(\Phi)$, the statement of Theorem 3.1 cannot be valid if we include elements $A$ of $\mathfrak{A}(\tilde{\mathcal{O}})$ with infinitely many non-zero $\left(A \mid \mathscr{F}_{m, n}\right)$ components. This can be easily verified for the case of the identity operator $\mathbf{1} \in \mathfrak{A}(\tilde{\mathcal{O}})$, since in that case the left-hand side of (3.3) is identically equal to $E_{ \pm}(\Phi)$ $-|\Phi\rangle\langle\Phi|$, while the right-hand side is $e^{-\Lambda(\Phi)}\left(E_{ \pm}(\Phi)-|\Phi\rangle\langle\Phi|\right)$.

Theorem 3.2. Let $\varrho(k, \sigma, v), v=-1,+1$, be two non-negative and essentially bounded,

$$
b_{v}=\underset{\sigma \in \mathbb{R}^{1}, \boldsymbol{k} \in \mathbb{R}^{3}}{\operatorname{ess}} \varrho(\boldsymbol{k}, \sigma, v)<\infty,
$$

Borel measurable functions on $\mathbb{R}^{4}$. Write

$$
\Delta_{v}^{r l}=S_{M}^{l} \cap\left\{(\boldsymbol{k}, \sigma): \frac{(r-1) b_{v}}{M}<\varrho(\boldsymbol{k}, \sigma, v) \leqq \frac{r b_{v}}{M}\right\}, \quad r=1, \ldots, M,
$$


where $\left\{S_{M}^{l}: l=1,2, \ldots\right\}$ is any subdivision of $\mathbb{R}^{4}$ into disjoint Borel sets $S_{M}^{l}$ of diameter smaller than $M^{-1}$. If $\chi_{v}^{r l}$ denotes the characteristic function of $\Delta_{v}^{r l}$, then

$$
E_{m, n} \bar{P}_{\varrho}=s_{M \rightarrow \infty} \sum_{v=-1}^{+1} \sum_{r=1}^{M} \sum_{l}^{\prime} \frac{1}{\mu\left(\Delta_{v}^{r l}\right)} a_{v}^{*}\left(\varrho \chi_{v}^{r l}\right) a_{v}\left(\chi_{v}^{r l}\right) E_{m, n}
$$

where the prime next to the summation sign indicates that the terms for which $\mu\left(\Delta_{v}^{r l}\right)=0$ should be omitted. Furthermore, there is a constant $C(m, n)$ independent of $M$ such that

$$
\left\|E_{m, n}\left(\bar{P}_{\varrho}-\sum_{v=-1}^{+1} \sum_{r=1}^{M} \sum_{l}^{\prime} \frac{1}{\mu\left(\Delta_{v}^{r l}\right)} a_{v}^{*}\left(\varrho \chi_{v}^{r l}\right) a_{v}\left(\chi_{v}^{r l}\right)\right)\right\| \leqq C(m, n) .
$$

Proof. We easily compute that for arbitrary $\Psi_{m, n} \in \mathscr{F}_{m, n}$

$$
\begin{aligned}
& a_{v}^{*}\left(\varrho \chi_{v}^{r l}\right) a_{v}\left(\chi_{v}^{r l}\right) \Psi_{m, n}\left(\boldsymbol{k}_{1}, \sigma_{1}, \ldots, \boldsymbol{k}_{m+n}, \sigma_{m+n}\right) \\
& \quad=\sum_{j=1}^{m+n} \delta(v, j) \varrho\left(\boldsymbol{k}_{j}, \sigma_{j}, v\right) \int_{\Delta_{v}^{l}} \Psi_{m}\left(\boldsymbol{k}_{1}, \sigma_{1}, \ldots \boldsymbol{k}_{j}^{\prime}, \sigma_{j}^{\prime}, \ldots \boldsymbol{k}_{m+n}, \sigma_{m+n}\right) d \mu\left(\boldsymbol{k}_{j}^{\prime}, \sigma_{j}^{\prime}\right)
\end{aligned}
$$

where $\delta(v, j)$ is zero for $j \leqq m, v=-1$ and for $j \geqq m+1, v=+1$, and it is one otherwise. Applying this result to

$$
Q_{M}=\sum_{v=-1}^{+1} \sum_{r=1}^{M} \sum_{l}^{\prime} \frac{1}{\mu\left(\Delta_{v}^{r l}\right)} a_{v}^{*}\left(\varrho \chi_{v}^{r l}\right) a_{v}\left(\chi_{v}^{r l}\right)
$$

we can easily see that for any simple function $\Phi_{m, n} \in \mathscr{F}_{m, n}$ built from characteristic functions of intervals in $\mathbb{R}^{4(m+n)}$ and for arbitrarily chosen constants $C_{v}^{r}$

$$
\begin{aligned}
& \left|\left\langle\Phi_{m, n} \mid E_{m, n}\left\{\bar{P}_{\varrho}-Q_{M}\right\} \Psi_{m, n}\right\rangle\right| \\
& \leqq \sum_{\nu=-1}^{+1} \sum_{j=1}^{m+n} \delta(v, j)\left\{\sum_{r=1}^{M} \sum_{l}^{\prime} \int_{\mathbb{R}^{4}(m+n)} d \mu\left(\boldsymbol{k}_{1}, \sigma_{1}\right) \ldots d \mu\left(\boldsymbol{k}_{m+n}, \sigma_{m+n}\right)\right. \\
& \times \chi_{v}^{r l}\left(\boldsymbol{k}_{j}, \sigma_{j}\right)\left\{\varrho\left(\boldsymbol{k}_{j}, \sigma_{j}, v\right)-C_{v}^{r} \mid \Phi_{m, n}^{*}\left(\boldsymbol{k}_{1}, \sigma_{1}, \ldots, \boldsymbol{k}_{m+n}, \sigma_{m+n}\right)\right. \\
& \times\left[\Psi_{m, n}\left(\boldsymbol{k}_{1}, \sigma_{1}, \ldots, \boldsymbol{k}_{m+n}, \sigma_{m+n}\right)\right. \\
& \left.-\frac{1}{\mu\left(\Delta_{v}^{r l}\right)} \int_{\Delta_{v}^{r l}} \Psi_{m, n}\left(\boldsymbol{k}_{1}, \sigma_{1}, \ldots, \boldsymbol{k}_{j}^{\prime}, \sigma_{j}^{\prime}, \ldots, \boldsymbol{k}_{m+n}, \sigma_{m+n}\right) d \mu\left(\boldsymbol{k}_{j}^{\prime}, \sigma_{j}^{\prime}\right)\right] \\
& +\sum_{r, l}^{\prime \prime}\left|C_{v}^{r}\right| \int_{\mathbb{R}^{4}(m+n)} \chi_{v}^{r l}\left(\boldsymbol{k}_{j}, \sigma_{j}\right) \mid \Phi_{m, n}\left(\boldsymbol{k}_{1}, \sigma_{1}, \ldots, \boldsymbol{k}_{m+n}, \sigma_{m+n}\right) \\
& \left.\times \Psi_{m, n}^{r, l}\left(\boldsymbol{k}_{1}, \sigma_{1}, \ldots, \boldsymbol{k}_{m+n}, \sigma_{m+n}\right) \mid d \mu\left(\boldsymbol{k}_{1}, \sigma_{1}\right) \ldots d \mu\left(\boldsymbol{k}_{m+n}, \sigma_{m+n}\right)\right\}
\end{aligned}
$$


where the summation $\Sigma^{\prime \prime}$ does not include any of the sets $\Delta_{v}^{r l}$ in which $\Phi_{m, n}^{*}$ is almost everywhere constant in $\left(k_{j} \sigma_{j}\right) \in \Delta_{v}^{r l}$ when all the other variables are kept fixed. If we take $C_{v}^{r}=\frac{(r-1) b_{v}}{M}$ then

$$
\varrho\left(\boldsymbol{k}_{j} \cdot \sigma_{j}, v\right)-C_{v}^{r} \leqq \frac{b_{v}}{M}, \quad\left(\boldsymbol{k}_{j}, \sigma_{j}\right) \in \Delta_{v}^{r l},
$$

and we get the estimate:

$$
\begin{aligned}
& \left|\left\langle\Phi_{m n} \mid E_{m, n}\left\{\bar{P}_{\varrho}-Q_{M}\right\} \Psi_{m, n}\right\rangle\right| \leqq \frac{2}{M} \sum_{v=-1}^{+1} b_{v} \sum_{j=1}^{m+n} \int_{\mathbb{R}^{4(m+n)}}\left|\Phi_{m, n}^{*} \Psi_{m n}\right| d \mu^{\times(m+n)} \\
& \quad+\sum_{j=1}^{m+n} \max _{r, v}\left|C_{v}^{r}\right|\left\|\Phi_{m, n}\right\|\left\{\left(\sum_{r, l}^{\prime \prime} \int \chi_{v}^{r l}\left(\boldsymbol{k}_{j}, \sigma_{j}\right)\left|\Psi_{m, n}\right|\right)^{2} d \mu^{\times(m+n)}\right\}^{\frac{1}{2}} \\
& \quad \leqq\left\|\Phi_{m, n}\right\|\left[2(m+n) \frac{\left(b_{-1}+b_{+1}\right)}{M}\left\|\Psi_{m, n}\right\|\right. \\
& \left.\quad+\left(b_{-1}+b_{+1}\right) \sum_{j=1}^{m+n}\left\{\sum_{r, l}^{\prime \prime} \int \chi_{v}^{r l}\left(\boldsymbol{k}_{j}, \sigma_{j}\right)\left|\Psi_{m, n}\right|^{2} d \mu^{\times(m+n)}\right\}^{\frac{1}{2}}\right]
\end{aligned}
$$

Since the family of all simple functions of $\mathscr{F}_{m, n}$ is dense in $\mathscr{F}_{m, n}$, we conclude from the above inequality that $\left\|E_{m, n}\left(\bar{P}_{\varrho}-Q_{M}\right) \Psi_{m, n}\right\|$ is not larger than the expression in the square bracket on the right-hand side of (3.9). In the limit $M \rightarrow \infty$ the measure $\sum_{r, l}^{\prime \prime} \mu\left(\Delta_{v}^{r l}\right)$ tends to zero, and therefore the aforementioned expression in (3.9) also converges to zero. Since $\Psi_{m, n}$ was an arbitrary vector in $\mathscr{F}_{m, n}$ and the family of all simple functions $\Phi_{m, n}$ built from characteristic functions of intervals on $\mathbb{R}^{4(m+n)}$ is dense in $\mathscr{F}_{m, n}$, this establishes that (3.6) is true.

To prove (3.7) note that the right-hand side of the inequality (3.9) is bounded by

$$
\left\|\Phi_{m, n}\right\|\left|\frac{2}{M}+1\right|(m+n)\left(b_{-1}+b_{+1}\right)\left\|\Psi_{m, n}\right\| .
$$

Consequently, $C(m, n)$ can be chosen to be any constant larger than $3(m+n)\left(b_{-1}+b_{+1}\right)$. Thus we have established Theorem 3.2 completely.

Let us remark that the proof of Theorem 3.1 would be simpler than it actually turns out to be if the limit in (3.6) were a uniform limit rather than a strong limit. However, it is quite easy to see that the convergence in (3.6) is not in operator bound, since the term $\Sigma^{\prime \prime}$ in the procedure cannot be made arbitrarily small independently of the chosen $\Phi_{m, n}$. Simple examples can be constructed where for given $\varepsilon>0, \Psi_{m, n}$ and $M$, that term exceeds $\varepsilon$ for some normalized $\Phi_{m, n}$.

In order to complete the proof of Theorem 3.1, we have to know the asymptotic behaviour in time of $U_{t} E_{m, n} U_{t}^{*}$. This is essentially derived in the following two lemmas. 
Lemma 3.1. For any eigenvector $\Phi$ of $H_{\kappa, v}$

$$
\underset{t \rightarrow \mp \infty}{w-\lim _{ \pm}} E_{ \pm}(\Phi) U_{t} E_{0,0} U_{t}^{*} E_{ \pm}(\Phi)=e^{-\Lambda(\Phi)} \Omega_{ \pm}(\Phi) E_{0,0} \Omega_{ \pm}^{*}(\Phi)
$$

where $\Lambda(\Phi)$ is the wave-amplitude renormalization constant defined in (3.2), and $E_{0,0}=\left|\Phi_{0,0}\right\rangle\left\langle\Phi_{0,0}\right|$.

Proof. According to (2.7), the restriction of $H_{\kappa, v}$ to $\mathscr{F}_{ \pm}(\Phi)$ has the same spectrum as $H_{0}+\eta \mathbf{1}$ has on $\mathscr{F}$. This spectrum obviously consists of a point spectrum which contains only the eigenvalue $\eta$ corresponding to $\Phi$, and of the absolutely continuous spectrum $\left[m_{0}+\eta,+\infty\right)$.

An arbitrary vector $\Psi$ from $\mathscr{F}_{ \pm}(\Phi)$ can be uniquely decomposed into the sum $\Psi^{(\eta)}+\Psi^{\text {a.c. }}$, where

$$
\Psi^{(\eta)}=\langle\Phi \mid \Psi\rangle \Phi
$$

and $\Psi^{\text {a.c. }}$ belongs to the absolutely continuous subspace of the restriction $\left(H_{\kappa, v} \mid \mathscr{F}_{ \pm}(\Phi)\right)$. This implies that for any $\Psi_{1} \in \mathscr{F}$ the Radon-Nikodym derivative $h(\lambda)$ of $\left\langle\Psi_{1} \mid E_{\lambda}^{H_{\kappa, v}} \Psi^{\text {a.c. }}\right\rangle$ with respect to the Lebesgue measure exists. Hence, by the Riemann-Lebesgue lemma,

$$
\begin{aligned}
\left\langle\Psi_{1} \mid e^{-i H_{\kappa, v} t} \Psi^{\text {a.c. }}\right\rangle & =\int_{-\infty}^{+\infty} e^{-i \lambda t} d\left\langle\Psi_{1} \mid E^{H_{\kappa, \nu}} \Psi^{\text {a.c. }}\right\rangle \\
& =\int_{-\infty}^{\infty} e^{-i \lambda t} h(\lambda) d \lambda
\end{aligned}
$$

converges to zero where $t \rightarrow \mp \infty$. Thus for any $\Psi_{1} \in \mathscr{F}_{ \pm}(\Phi)$

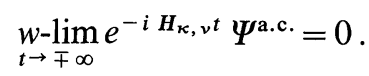

To establish (3.10), note that by carrying out the above decomposition for arbitrary $\Psi_{1}, \Psi_{2} \in \mathscr{F}_{ \pm}(\Phi)$ we obtain

$$
\begin{gathered}
\left\langle\Psi_{1} \mid U_{t} E_{0,0} U_{t}^{*} \Psi_{2}\right\rangle=\left\langle\Psi_{1} \mid \Phi\right\rangle\left|\left\langle\Phi \mid \Phi_{0,0}\right\rangle\right|^{2}\left\langle\Phi \mid \Psi_{2}\right\rangle \\
+e^{i \eta t}\left\langle\Psi_{1} \mid \Phi\right\rangle\left\langle\Phi \mid \Phi_{0,0}\right\rangle\left\langle\Phi_{0,0} \mid e^{-i H_{\kappa, v} t} \Psi_{2}^{\text {a.c.c. }}\right\rangle \\
+e^{-i \eta t}\left\langle e^{-i H_{\kappa, v} t} \Psi_{1}^{\text {a.c. }} \cdot \mid \Phi_{0,0}\right\rangle\left\langle\Phi_{0,0} \mid \Phi\right\rangle\left\langle\Phi \mid \Psi_{2}\right\rangle \\
+\left\langle e^{-i H_{\kappa, v} t} \Psi_{1}^{\text {a.c. }} \mid \Phi_{0,0}\right\rangle\left\langle\Phi_{0,0} \mid e^{-i H_{\kappa, v} t} \Psi_{2}^{\text {a.c. }}\right\rangle
\end{gathered}
$$

Thus in view of (3.12), we can write

$$
\begin{aligned}
\lim _{t \rightarrow \mp \infty}\left\langle\Psi_{1} \mid U_{t} E_{0,0} U_{t}^{*} \Psi_{2}\right\rangle & =\left|\left\langle\Phi \mid \Phi_{0,0}\right\rangle\right|^{2}\left\langle\Psi_{1} \mid \Phi\right\rangle\left\langle\Phi \mid \Psi_{2}\right\rangle \\
& =e^{-\Lambda(\Phi)}\left\langle\Psi_{1} \mid \Omega_{ \pm} E_{0,0} \Omega^{*} \Psi_{2}\right\rangle
\end{aligned}
$$

which is the required result (3.10). 
Now we want to extend the result of Lemma 3.1 to the projector $E_{m, n}$ for $m+n>0$, with $E_{ \pm}(\Phi)$ replaced by $E_{ \pm}(\Phi)-|\Phi\rangle\langle\Phi|$. To do this, note that if $h_{1}, h_{2}, \ldots$ is an arbitrary orthonormal basis in $L_{\mu}^{2}\left(\mathbb{R}^{4}\right)$, then $E_{m, n}$ can be written in the form

$$
\begin{aligned}
E_{m, n}= & \sum_{p_{1}, \ldots, p_{m+n}=1}^{\infty} a_{1}^{*}\left(\bar{h}_{p_{1}}\right) \ldots a_{1}^{*}\left(\bar{h}_{p_{m}}\right) a_{-1}^{*}\left(\bar{h}_{p_{m+1}}\right) \ldots a_{-1}^{*}\left(\bar{h}_{p_{m+n}}\right) E_{0,0} \\
& \cdot a_{-1}\left(h_{p_{m+n}}\right) \ldots a_{-1}\left(h_{p_{m+1}}\right) a_{1}\left(h_{p_{m}}\right) \ldots a_{1}\left(h_{p_{1}}\right)
\end{aligned}
$$

where the infinite sum is the strong limit of its finite partial sums. It is easy to see from Lemma 3.1 and (2.9) that for each term in (3.14) we have

$$
\begin{gathered}
\underset{\substack{w \rightarrow \mp \infty \\
t \rightarrow \lim _{ \pm}}}{ } E_{ \pm}(\Phi) U_{t} a_{1}^{*}\left(\bar{h}_{i_{1}}\right) \ldots a_{-1}^{*}\left(\bar{h}_{j_{m}}\right) E_{0,0} a_{-1}\left(h_{j_{m}}\right) \ldots a_{1}\left(h_{i_{1}}\right) U_{t}^{*} E_{ \pm}(\Phi) \\
\quad=e^{-\Lambda(\Phi)} \Omega_{ \pm}(\Phi) a_{1}^{*}\left(\bar{h}_{i_{1}}\right) \ldots a_{-1}^{*}\left(\bar{h}_{j_{m}}\right) E_{0,0} a_{-1}\left(h_{j_{m}}\right) \ldots a_{1}\left(h_{i_{1}}\right) \Omega_{ \pm}^{*}(\Phi) .
\end{gathered}
$$

For example for $m=1, n=0$ we have

$$
\begin{aligned}
& E_{ \pm} U_{t} a^{*}(\bar{h}) E_{0,0} a(h) U_{t}^{*} E_{ \pm} \\
& =E_{ \pm} U_{t} a^{*}(\bar{h}) U_{t}^{*}\left[E_{ \pm}+\left(1-E_{ \pm}\right)\right] U_{t} E_{0,0} U_{t}^{*}\left[E_{ \pm}+\left(1-E_{ \pm}\right)\right] U_{t} a(h) U_{t}^{*} E_{ \pm}
\end{aligned}
$$

and the desired result is a consequence of the facts that the existence of the weak limit of a product follows from the existence of the weak limit of one of its factors and of the uniform limit of the rest of its factors, and that if one factor converges uniformly to zero and the rest of the factors stay bounded uniformly in $t$ then the uniform limit is zero.

Now we note that since $[3,5,6]$

$$
a_{ \pm 1}(h) \Phi=0
$$

it follows that (3.15) is also true if we replace $E_{ \pm}(\Phi)$ by $\left\{E_{ \pm}(\Phi)-|\Phi\rangle\langle\Phi|\right\}$. Therefore, each term in the summation in (3.14) satisfies Lemma 3.1 with $E_{ \pm}(\Phi)$ replaced by $\left\{E_{ \pm}(\Phi)-|\Phi\rangle\langle\Phi|\right\}$.

After proving the next lemma it will become easy to see that $E_{m, n}$, $m+n>0$, also satisfies Lemma 3.1 when we replace $E_{ \pm}(\Phi)$ by

$$
\left\{E_{ \pm}(\Phi)-|\Phi\rangle\langle\Phi|\right\}
$$

in (3.10).

Lemma 3.2. For any $\Psi \in \mathscr{F}_{ \pm}(\Phi)$ orthogonal to $\Phi$ and for any $\varepsilon>0$, there exists an $N(\varepsilon)$ and $T(\varepsilon)$ such that

$$
\sum_{p_{1}+\cdots+p_{m+n} \geqq N(\varepsilon)}\left|\left\langle a_{1}^{*}\left(h_{p_{1}}\right) \ldots a_{-1}^{*}\left(h_{p_{m+n}}\right) \Phi_{0,0} \mid U_{t}^{*} \Psi\right\rangle\right|^{2}<\varepsilon
$$

for all $|t| \geqq T(\varepsilon)$. 
Proof. We shall prove (3.16) explicitly only for $m=1, n=0$, i.e., prove that

$$
\sum_{p \geqq N(\varepsilon)}\left|\left\langle a_{1}^{*}\left(h_{p}\right) \Phi_{0,0} \mid U_{t}^{*} \Psi\right\rangle\right|^{2}<\varepsilon
$$

for all $|t| \geqq T(\varepsilon)$ and $\Psi \in\left\{E_{ \pm}(\Phi)-|\Phi\rangle\langle\Phi|\right\} \mathscr{F}$.

Differentiating and then integrating in $t$ each term in (3.17), we obtain that the sum in (3.17) is equal to

$$
\begin{aligned}
& \sum_{p \geqq N(\varepsilon)}\left|\left\langle a_{1}^{*}\left(h_{p}\right) \Phi_{0,0} \mid U_{ \pm T(\varepsilon)}^{*} \Psi\right\rangle\right|^{2} \\
& \quad+\sum_{p \geqq N(\varepsilon)} \int_{ \pm T(\varepsilon)}^{t} \frac{d}{d t^{\prime}}\left|\left\langle a_{1}^{*}\left(h_{p}\right) \Phi_{0,0} \mid U_{t^{\prime}}^{*} \Psi\right\rangle\right|^{2} d t^{\prime} .
\end{aligned}
$$

The second term in the expression above is equal to

$$
\begin{aligned}
& \sum_{p \geqq N(\varepsilon)} \int_{ \pm T(\varepsilon)}^{t} \\
& \cdot d t^{\prime}\left\langle\Psi \mid e^{i t^{\prime} H_{\kappa, v}}\left[H_{I, \kappa, v}, a_{1}^{*}\left(e^{-i \omega t^{\prime}} h_{p}\right) E_{0,0}, a_{1}\left(e^{i \omega t^{\prime}} h_{p}\right)\right]_{-} e^{-i t^{\prime} H_{\kappa, v}} \Psi\right\rangle .
\end{aligned}
$$

Using the notation in Ref. [3], we may write

$$
\left[H_{I, \kappa, v}, a\left(e^{i \omega t} h_{p}\right)\right]_{-}=\sum_{i} \sum_{j=1}^{l_{i}}(-1)^{j-1} \delta_{v v_{j}} \int_{\mathbb{R}^{3}} v(\boldsymbol{x}) V_{j}^{\prime}(\boldsymbol{x}) \hat{h}_{p \alpha_{j}}(\boldsymbol{x}, t) d^{3} \boldsymbol{x} .
$$

Hence, by applying the Schwarz-Cauchy inequality in $l^{2}(\infty)$ and observing that

$$
\begin{aligned}
\sum_{p=1}^{\infty}\left|\left\langle e^{-i t H_{\kappa, \nu}} \Psi \mid a_{1}^{*}\left(e^{-i \omega t} h_{p}\right) \Phi_{0,0}\right\rangle\right|^{2} & =\left\|E_{1,0} e^{i H_{0} t} e^{-i t H_{\kappa, \nu}} \Psi\right\|^{2} \\
& =\left\|E_{1,0} e^{-i t H_{\kappa, \nu}} \Psi\right\|^{2} \\
& \leqq\|\Psi\|^{2}
\end{aligned}
$$

and in view of the fact that for any $\Psi^{\prime}, \Psi^{\prime \prime} \in \mathscr{F}$

$$
\begin{aligned}
& \left\langle\Psi^{\prime} \mid\left[H_{I, \kappa, v}, a_{1}^{*}\left(e^{-i \omega t} h_{p}\right) E_{0,0} a_{1}\left(e^{i \omega t} h_{p}\right)\right]_{-} \Psi^{\prime \prime}\right\rangle \\
& \quad=\left\langle a_{1}^{*}\left(e^{-i \omega t} h_{p}\right) \Phi_{0,0} \mid \Psi^{\prime \prime}\right\rangle\left\langle\Psi^{\prime} \mid\left[H_{I, \kappa, v}, a_{1}^{*}\left(e^{-i \omega t} h_{p}\right)\right]_{-} \Phi_{0,0}\right\rangle \\
& \quad+\left\langle\Psi^{\prime} \mid a_{1}^{*}\left(e^{-i \omega t} h_{p}\right) \Phi_{0,0}\right\rangle\left\langle\Phi_{0,0} \mid\left[H_{I, \kappa, v}, a_{1}\left(e^{i \omega t} h_{p}\right)\right]_{-} \Psi^{\prime \prime}\right\rangle
\end{aligned}
$$

we get that (3.19) is in absolute value bounded by

$$
\begin{aligned}
& \int_{ \pm T(\varepsilon)}^{t} d t^{\prime}\|\Psi\| \sum_{i} \sum_{j=1}^{l_{i}} \\
& \cdot \delta_{v v_{j}}\left(\sum_{p=1}^{\infty}\left|\left\langle\Phi_{0,0} \mid \int_{\mathbb{R}^{3}} v(\boldsymbol{x}) V_{j}^{\prime}(\boldsymbol{x}) \hat{h}_{p \alpha_{j}}\left(\boldsymbol{x}^{\prime}, t\right) d^{3} \boldsymbol{x} e^{-i t^{\prime} \boldsymbol{H}_{\kappa, v}} \Psi\right\rangle\right|^{2}\right)^{\frac{1}{2}}
\end{aligned}
$$

for all $N(\varepsilon)$. 
Now let us write

and

$$
v(\boldsymbol{x})\left\langle\Phi_{0,0} \mid V_{j}^{\prime}(\boldsymbol{x}) e^{-i t H_{\kappa, v}} \Psi\right\rangle=F(\boldsymbol{x}, t)
$$

$$
\tilde{F}(\boldsymbol{k}, t)=(2 \pi)^{-\frac{3}{2}} \int_{\mathbb{R}^{3}} e^{-i \boldsymbol{k} \cdot \boldsymbol{x}} F(\boldsymbol{x}, t) d^{3} \boldsymbol{x} .
$$

Since $e^{-i \omega t} h_{1}, e^{-i \omega t} h_{2}, \ldots$ constitute an orthonormal basis in $L_{\mu}^{2}\left(\mathbb{R}^{3}\right)$, we easily see that

$$
\begin{aligned}
& \sum_{p=1}^{\infty}\left|\int_{\mathbb{R}^{3}} d \boldsymbol{x}\left\langle\Phi_{0,0} \mid v(\boldsymbol{x}) V_{j}^{\prime}(\boldsymbol{x}) e^{-i t H_{\kappa, v}} \Psi\right\rangle \hat{h}_{p \alpha_{j}}(\boldsymbol{x}, t)\right|^{2} \\
& \quad=\sum_{p=1}^{\infty}\left|\int_{\mathbb{R}^{3}} \omega(\boldsymbol{k}) \tilde{F}(\boldsymbol{k}, t) \beta_{\alpha_{j}}(\boldsymbol{k}, \sigma) e^{-i \omega(\boldsymbol{k}) t} h_{p}\left(\boldsymbol{k}, \sigma_{j}\right) \frac{d \boldsymbol{k}}{\omega(\boldsymbol{k})}\right|^{2} \\
& \quad=\int_{\mathbb{R}^{3}} \omega(\boldsymbol{k})\left|\beta_{\alpha_{j}}(\boldsymbol{k}, \sigma) \tilde{F}(\boldsymbol{k}, t)\right|^{2} d \boldsymbol{k}
\end{aligned}
$$

where

$$
\hat{h}_{p_{\alpha_{j}}}(\boldsymbol{x}, t)=\int \beta_{\alpha_{j}}(\boldsymbol{k}, \sigma) h_{p}(\boldsymbol{k}, \sigma) e^{i[\omega(\boldsymbol{k}) t-\boldsymbol{k} \cdot \boldsymbol{x}]} d \mu(\boldsymbol{k}, \sigma) .
$$

Taking into consideration the fact that $\beta_{\alpha_{j}}(\boldsymbol{k}, \sigma)$ vanishes [3] for $|\boldsymbol{k}|>\kappa$, we obtain that (3.21) is bounded by

$$
\|\Psi\| \sum_{i} \sum_{j=1}^{l_{i}} \delta_{v v_{j}}\left[\omega(\kappa) \sup _{|\boldsymbol{k}| \leqq \kappa}\left|\beta_{\alpha_{j}}(\boldsymbol{k}, \sigma)\right|^{2}\right]^{\frac{1}{2}} \int_{ \pm T(\varepsilon)}^{t}\left[\int\left|\tilde{F}\left(\boldsymbol{k}, t^{\prime}\right)\right|^{2} d \boldsymbol{k}\right]^{\frac{1}{2}} d t^{\prime}
$$

where

$$
\int_{\mathbb{R}^{3}}|\tilde{F}(\boldsymbol{k}, t)|^{2} d \boldsymbol{k}=\int_{\mathbb{R}^{3}} v^{2}(\boldsymbol{x})\left|\left\langle V_{j}^{\prime *}(\boldsymbol{x}) \Phi_{0,0} \mid e^{-i t H_{\kappa, v}} \Psi\right\rangle\right|^{2} d \boldsymbol{x} .
$$

Consider now vectors $\Psi$ from the linear manifolds $\mathscr{P}_{ \pm}(\Phi)$ spanned by all vectors of the form

$$
a_{1}^{*}\left(f_{1}\right) \ldots a_{1}^{*}\left(f_{m}\right) a_{-1}^{*}\left(f_{m+1}\right) \ldots a_{-1}^{*}\left(f_{m+n}\right) \Phi
$$

for all $m+n \geqq 1$, and all $f_{1}, \ldots f_{m+n} \in \mathscr{C}_{0}^{\infty}\left(\mathbb{R}^{4}\right)$ which are such that

$$
\left\{\boldsymbol{k}: \boldsymbol{k}^{2}=\kappa^{2}\right\} \cap \sup f_{l}(\boldsymbol{k}, \sigma)=\emptyset .
$$

It is quite evident that $\mathscr{P}_{ \pm}(\Phi)$ is dense in $\mathscr{F}_{ \pm}(\Phi) \ominus[\Phi]$.

All the vectors $\Psi \in \mathscr{P}_{ \pm}(\Phi)$ obviously belong to the absolutely continuous spectrum of the restriction of $H_{\kappa, v}$ to $\mathscr{F}_{ \pm}(\Phi)$, and for each such $\Psi$ there is an $\lambda_{\Psi}$ such that $E^{H_{\kappa}, v} \Psi=\Psi$ for $\lambda \geqq \lambda_{\Psi}$. Hence, employing the spectral theorem and denoting by $\phi_{\Psi}(\lambda, x)$ the Radon-Nikodyn derivative of $\left\langle V_{j}^{\prime *}(x) \Phi_{0,0} \mid E_{\lambda}^{H_{\kappa, v}} \Psi\right\rangle$ with respect to the Lebesgue measure, we can 
write (3.23) in the form

$$
\begin{aligned}
\int_{\mathbb{R}^{3}} d x & v^{2}(x) \int_{0}^{\lambda_{\Psi}} e^{i \lambda_{1} t} \phi_{\Psi}\left(\lambda_{1}, x\right) d \lambda_{1} \int_{0}^{\lambda_{\Psi}} e^{-i \lambda_{2} t} \phi_{\Psi}^{*}\left(\lambda_{2}, x\right) d \lambda_{2} \\
= & \int_{-\lambda_{\Psi}}^{\lambda_{\Psi}} e^{i \lambda^{\prime} t} \tau_{\Psi}\left(\lambda^{\prime}\right) d \lambda^{\prime}=\tilde{\tau}_{\Psi}(t)
\end{aligned}
$$

where

$$
\tau_{\Psi}\left(\lambda^{\prime}\right)=\int_{\mathbb{R}^{4}} \phi_{\Psi}^{*}\left(\frac{\lambda^{\prime \prime}-\lambda^{\prime}}{2}, \boldsymbol{x}\right) \phi_{\Psi}\left(\frac{\lambda^{\prime \prime}+\lambda^{\prime}}{2}, \boldsymbol{x}\right) v^{2}(\boldsymbol{x}) d \lambda^{\prime \prime} d \boldsymbol{x}
$$

The interchange of order of integration required in deriving (3.26) can be easily justified by Fubini's theorem, since we are integrating on compact domains.

The assertion that the expression in (3.21) can be made smaller than any $\varepsilon>0$ if $T(\varepsilon)$ is chosen appropriately large, becomes an obvious truth if $\sqrt{\tilde{\tau}_{\Psi}(t)}$ is integrable in $t$ on $\mathbb{R}^{1}$. Since $\tau_{\Psi}(t)$ is evidently continuous, we infer that $\sqrt{\tilde{\tau}_{\Psi}(t)}$ is integrable on $\mathbb{R}^{1}$ if $\tau_{\psi}(t)$ decreases at infinity faster than $|t|^{-3}$, i.e., if $\tau_{\Psi}(\lambda)$ is at least three times differentiable. We verify that this is always so when $\Psi \in \mathscr{P}_{ \pm}(\Phi)$ for the special case $\Psi=a_{v \pm}^{*}(f) \Phi$. The general case can be treated in the same manner.

By definition, for such $\Psi$

$$
\phi_{\Psi}(\lambda, \boldsymbol{x})=\frac{d}{d \lambda}\left\langle V_{j}^{\prime *}(\boldsymbol{x}) \Phi_{0,0} \mid a_{v \pm}^{*}\left(f_{\lambda}\right) \Phi\right\rangle
$$

where $f_{\lambda}(\boldsymbol{k}, \sigma)=f(\boldsymbol{k}, \sigma)$ when $\omega(\boldsymbol{k}) \leqq \lambda$, and $f_{\lambda}(\boldsymbol{k}, \sigma)=0$ when $\omega(\boldsymbol{k})>\lambda$.

$$
\begin{aligned}
& \left\langle V_{j}^{\prime *}(\boldsymbol{x}) \Phi_{0,0} \mid a_{v \pm}^{*}\left(f_{\lambda}\right) \Psi\right\rangle=\left\langle V_{j}^{\prime *}(\boldsymbol{x}) \Phi_{0,0} \mid a_{v}^{*}\left(f_{\lambda}\right) \Phi\right\rangle \\
& \quad+i \int_{0}^{\mp \infty}\left\langle e^{-i t H_{\kappa, v}} V_{j}^{\prime *}(\boldsymbol{x}) \Phi_{0,0} \mid\left[H_{I, \kappa, v}, a^{*}\left(e^{-i \omega t} f_{\lambda}\right)\right] \Phi\right\rangle e^{-i \eta t} d t,
\end{aligned}
$$

the second term on the right-hand side of the above relation may be written as

$$
\begin{aligned}
& \sum_{j^{\prime}=1}^{l} i(-1)^{j^{\prime}-1} \delta_{v v_{j}} \int_{0}^{\mp \infty} d t \int_{\mathbb{R}^{3}} d \boldsymbol{x}^{\prime}\left\langle e^{-i t H_{\kappa}, v} V_{j^{\prime}}^{\prime *}(\boldsymbol{x}) \Phi_{0,0} \mid V_{j^{\prime}}^{\prime}\left(\boldsymbol{x}^{\prime}\right) \Phi\right\rangle \\
& \cdot v(\boldsymbol{x}) \int_{|\boldsymbol{k}| \leqq \kappa} e^{i(\omega(\boldsymbol{k}) t-\boldsymbol{k} \boldsymbol{x})} \beta_{\alpha_{j}}(\boldsymbol{k}, \sigma) f_{\lambda}(\boldsymbol{k}, \sigma) d \mu(\boldsymbol{k}, \sigma) .
\end{aligned}
$$

Taking into account (3.25) and the fact that $f(\boldsymbol{k},.) \in \mathscr{C}_{0}^{\infty}\left(\mathbb{R}^{3}\right)$, as well as the uniform convergence with respect to $t$ of the above improper Riemann integral, we easily infer that the expression in (3.28) is differentiable any number of times with respect $\lambda$, and therefore $\tilde{\tau}_{\Psi}(t)=0\left(|t|^{-r}\right)$ for any $r=1,2, \ldots$. 
The above considerations establish the fact that for any given $\Psi \in \mathscr{P}_{ \pm}(\Phi)$ and any $\varepsilon>0$ there is a $T(\varepsilon)$ for which the sum in (3.19) is in absolute value smaller than $\varepsilon / 2$ for all $|t|>T(\varepsilon)$ and for arbitrary $N(\varepsilon)$. From this result and (3.18) we easily deduce that (3.17) holds if for fixed $T(\varepsilon)$ we choose $N(\varepsilon)$ in such a manner that the first sum in (3.18) is smaller than $\varepsilon / 2$. Hence, (3.17) holds for $\Psi \in \mathscr{P}_{ \pm}(\Phi)$. Since $\mathscr{P}_{ \pm}(\Phi)$ is dense $\mathscr{F}_{ \pm}(\Phi) \ominus[\Phi]$, the result can be immediately extended to arbitrary $\Psi \in \mathscr{F}_{ \pm}(\Phi)$ orthogonal to $\Phi$.

This completes the proof of Lemma 3.2 for $E_{1,0}$. As was earlier mentioned, the proof for the general case $E_{m, n}$ runs along the same lines.

In view of (3.15) and the remarks preceding Lemma 3.2, we can infer from Lemma 3.2 that

$$
\begin{aligned}
& \underset{t \rightarrow \mp \infty}{w-\lim _{ \pm}}\left(E_{ \pm}(\Phi)-|\Phi\rangle\langle\Phi|\right) U_{t} E_{m, n} U_{t}^{*}\left(E_{ \pm}(\Phi)-|\Phi\rangle\langle\Phi|\right) \\
& =e^{-\Lambda}\left(E_{ \pm}(\Phi)-|\Phi\rangle\langle\Phi|\right) \Omega_{ \pm}(\Phi) E_{m, n} \Omega_{ \pm}^{*}(\Phi)\left(E_{ \pm}(\Phi)-|\Phi\rangle\langle\Phi|\right) .
\end{aligned}
$$

Now we shall return to proving Theorem 3.1 for an element of $\mathfrak{A}_{0}(\tilde{\mathcal{O}})$ of the form $A=E_{m, n} \bar{P}_{\varrho_{1}} \ldots \bar{P}_{\varrho_{l}}$. First, we observe that the case $m=n=0$ is immediately taken care of since in that case both sides of (3.3) vanish identically. Hence, let us take $m+n>0$ and, assuming that

$$
E_{m, n} \bar{P}_{\varrho_{j}}=\underset{M \rightarrow \infty}{s-\lim } Q_{M}^{(j)} E_{m, n}, \quad j=1, \ldots, l,
$$

let us consider the identity

$$
\begin{aligned}
& E_{ \pm}^{\prime} U_{t} E_{m, n} \bar{P}_{\varrho_{1}} \ldots \bar{P}_{\varrho_{l}} U_{t}^{*} E_{ \pm}^{\prime}-\Omega_{ \pm} E_{m, n} \bar{P}_{\varrho_{1}} \ldots \bar{P}_{\varrho_{l}} \Omega_{ \pm}^{*} E_{ \pm}^{\prime} e^{-\Lambda} \\
& \quad=R_{1}(M)+R_{2}(M, t)+R_{3}(M, t)
\end{aligned}
$$

where $E_{ \pm}^{\prime}=E_{ \pm}(\Phi)-|\Phi\rangle\langle\Phi|$ and

$$
\begin{gathered}
R_{1}(M)=-e^{-\Lambda} E_{ \pm}^{\prime} \Omega_{ \pm} E_{m, n}\left(\bar{P}_{\varrho_{1}} \ldots \bar{P}_{\varrho_{l}}-Q_{M}^{(1)} \ldots Q_{M}^{(l)}\right) \Omega_{ \pm}^{*} E_{ \pm}^{\prime}, \\
R_{2}(M, t)=E_{ \pm}^{\prime}\left(U_{t} E_{m, n} Q_{M}^{(1)} \ldots Q_{M}^{(l)} U_{t}^{*}-e^{-\Lambda} \Omega_{ \pm} E_{m, n} Q_{M}^{(1)} \ldots Q_{M}^{(l)} \Omega_{ \pm}^{*}\right) E_{ \pm}^{\prime} \\
R_{3}(M, t)=E_{ \pm}^{\prime} U_{t} E_{m, n}\left[\bar{P}_{\varrho_{1}} \ldots \bar{P}_{\varrho_{l}}-Q_{M}^{(1)} \ldots Q_{M}^{(l)}\right] U_{t}^{*} E_{ \pm}^{\prime}
\end{gathered}
$$

In view of Theorem 3.2, $R_{1}(M)$ converges strongly to zero when $M \rightarrow \infty$. For fixed $M, R_{2}(M, t)$ converges weakly to zero when $t \rightarrow \mp \infty$, since (3.29) holds and from the form of each $Q_{M}^{(j)}$, as given in (3.8), we immediately infer by using (2.9) that

$$
\begin{aligned}
\underset{t \rightarrow \mp \infty}{w-\lim _{t}} U_{t} Q_{M}^{(1)} \ldots Q_{M}^{(l)} U_{t}^{*} E_{ \pm}^{\prime} & =\Omega_{ \pm} Q_{M}^{(1)} \ldots Q_{M}^{(l)} \Omega_{ \pm}^{*} E_{ \pm}^{\prime} \\
& =E_{ \pm}^{\prime} \Omega_{ \pm} Q_{M}^{(1)} \ldots Q_{M}^{(l)} \Omega_{ \pm}^{*} E_{ \pm}^{\prime}
\end{aligned}
$$


the last of the above relations is an immediate consequence of the fact that

$$
Q_{M}^{(1)} \ldots Q_{M}^{(l)} \Omega_{ \pm}^{*} \Phi=Q_{M}^{(1)} \ldots Q_{M}^{(l)} \Phi_{0,0}=\mathbf{0} .
$$

Thus in order to show that the expression in (3.31) converges weakly to zero when $t \rightarrow \mp \infty$, it is sufficient to show that for any $\Psi_{1}, \Psi_{2} \in \mathscr{F}_{ \pm}^{\prime}(\Phi)$ and any $\varepsilon>0$ there is a $T(\varepsilon)$ such that

$$
\left|\left\langle\Psi_{1} \mid R_{3}(M, t) \Psi_{2}\right\rangle\right|<\varepsilon
$$

for all $|t|>T(\varepsilon)$ and arbitrary $M=1,2, \ldots$. For that we need the following lemma.

Lemma 3.3. For given $\Psi_{1}, \Psi_{2} \in \mathscr{F}_{ \pm}^{\prime}(\Phi)$, given $E_{m, n}$, and any $\varepsilon>0$, there is $N(\varepsilon)$ and $T(\varepsilon)$ such that

$$
\begin{gathered}
\sum_{p_{1}+\cdots+p_{m+n}>N(\varepsilon)} \mid\left\langle\Psi_{1}\right| U_{t} E_{m, n}\left(\bar{P}_{\varrho_{1}} \ldots \bar{P}_{\varrho_{l}}-Q_{M}^{(1)} \ldots Q_{M}^{(l)}\right) \\
\left.\cdot a_{1}^{*}\left(\bar{h}_{1}\right) \ldots a_{-1}^{*}\left(\bar{h}_{p_{m+n}}\right) \Phi_{0,0}\right\rangle\left\langle\Phi_{0,0} \mid a_{1}\left(h_{p_{1}}\right) \ldots a_{-1}\left(h_{p_{m+n}}\right) U_{t}^{*} \Psi_{2}\right\rangle \mid
\end{gathered}
$$

is smaller than $\varepsilon$ for all $|t|>T(\varepsilon)$.

Proof. The proof is a straightforward application of Lemma 3.2. Using the Schwarz-Cauchy inequality in $l^{2}(\infty)$, we get that (3.36) is majorized by

$$
\begin{aligned}
& \left\|E_{m, n}\left(\bar{P}_{\varrho_{1}} \ldots \bar{P}_{\varrho_{l}}-Q_{M}^{(1)} \ldots Q_{M}^{(l)}\right) E_{m, n} U_{t} \Psi_{1}\right\| \\
& \left\{\sum_{p_{1}+\ldots+p_{m+n}>N(\varepsilon)}\left|\left\langle\Psi_{2} \mid U_{t_{1}}^{*} a_{1}^{*}\left(h_{p_{1}}\right) \ldots a_{1}^{*}\left(h_{p_{m+n}}\right) \Phi_{0,0}\right\rangle\right|^{2}\right\}^{\frac{1}{2}} .
\end{aligned}
$$

The first factor can be majorized by

$$
\left\|\left(\bar{P}_{\varrho_{1}} \ldots \bar{P}_{\varrho_{l}}-Q_{M}^{(1)} \ldots Q_{M}^{(l)}\right) E_{m, n}\right\|\left\|\Psi_{1}\right\|
$$

which is independent of $t$, and which, according to (3.7), can be majorized in its turn by a constant independent of $M$. The second factor in (3.37) can be made, by Lemma 3.2 , arbitrarily small by choosing $N(\varepsilon)$ sufficiently large. Hence Lemma 3.3 is established.

In order to establish (3.35), it only remains to observe that in view of (3.30)

$$
\begin{aligned}
& \sum_{p_{1}+\cdots+p_{m+n} \leqq N(\varepsilon)} \mid\left\langle\Psi_{1}\right| U_{t} E_{m, n}\left(\bar{P}_{\varrho_{1}} \ldots \bar{P}_{\varrho_{l}}-Q_{M}^{(1)} \ldots Q_{M}^{(l)}\right) \\
& \left.\quad \cdot a_{1}^{*}\left(\bar{h}_{1}\right) \ldots a_{-1}^{*}\left(\bar{h}_{p_{m+n}}\right) \Phi_{0,0}\right\rangle\left\langle\Phi_{0,0} \mid a_{1}\left(h_{p_{1}}\right) \ldots a_{-1}\left(h_{p_{m+n}}\right) U_{t}^{*} \Psi_{2}\right\rangle \mid \\
& \leqq \\
& \quad \sum_{p_{1}+\cdots+p_{m+n} \leqq N(\varepsilon)}\left\|\Psi_{1}\right\|\left\|\Psi_{2}\right\|\left\|a_{1}\left(h_{p_{1}}\right) \ldots a_{-1}\left(h_{p_{m+n}}\right)\right\| \\
& \quad\left\|E_{m, n}\left(\bar{P}_{\varrho_{1}} \ldots \bar{P}_{\varrho_{l}}-Q_{M}^{(1)} \ldots Q_{M}^{(l)}\right) a_{1}^{*}\left(\bar{h}_{1}\right) \ldots a_{-1}^{*}\left(\bar{h}_{p_{m+n}}\right) \Phi_{0,0}\right\|
\end{aligned}
$$

can be made arbitrarily small independently of $t$. 
Thus, the proof of Theorem 3.1 for elements of $\mathfrak{A}_{0}(\tilde{\mathcal{O}})$ of the form $E_{m, n} \bar{P}_{\ell_{1}} \ldots \bar{P}_{\ell_{l}}$ has been completed. Since any element of $\mathfrak{A}_{0}(\tilde{\mathcal{O}})$ is a finite linear combination of elements of this type, Theorem 3.1 has been established for all $A \in \mathfrak{U}_{0}(\tilde{\mathcal{O}})$.

Consider now any element $A$ of $\mathfrak{U}_{0}(\tilde{\mathcal{O}})$. By recapitulating the proof of Theorem 3.1 [3], we easily establish that the following statement is true (observe especially (3.12)).

Lemma 3.4. For any given $A \in \mathfrak{A}(\tilde{\mathcal{O}})$ and $E_{m, n}$ there is a sequence $A_{1}, A_{2}, \ldots \in \mathfrak{A}_{0}(\tilde{\mathcal{O}})$ such that

$$
A E_{m, n}=s-\lim _{l \rightarrow \infty} A_{l} E_{m, n}
$$

and a constant $C$ for which

$$
\left\|\left(A-A_{l}\right) E_{m, n}\right\| \leqq C, \quad l=1,2, \ldots .
$$

For any $\Psi_{1}, \Psi_{2} \in \mathscr{F}_{ \pm}^{\prime}(\Phi)$ we can write

$$
\begin{aligned}
& \left\langle\Psi_{1} \mid\left(U_{t} E_{m, n} A U_{t}^{*}-e^{-\Lambda} \Omega_{ \pm} E_{m, n} A \Omega_{ \pm}^{*}\right) \Psi_{2}\right\rangle \\
& \quad \leqq \\
& \quad \leqq \Psi_{1}\left|\Omega_{ \pm} E_{m, n}\left(A-A_{l}\right) \Omega_{ \pm}^{*} \Psi_{2}\right\rangle \mid \\
& \quad+\mid\left\langle\Psi_{1} \mid\left(U_{t} E_{m, n} A_{l} U_{t}^{*}-e^{-\Lambda} \Omega_{ \pm} E_{m, n} A_{l} \Omega_{ \pm}^{*}\right) \Psi_{2}\right\rangle \\
& \quad+R(l, t)
\end{aligned}
$$

where, in view of (3.39), $R(l, t)$ can be estimated in the following manner:

$$
\begin{aligned}
R(l, t)= & \left|\left\langle\Psi_{1} \mid U_{t} E_{m, n}\left(A-A_{l}\right) U_{t}^{*} \Psi_{2}\right\rangle\right| \\
& \leqq \sum_{p_{1}+\cdots+p_{m+n} \leqq K}\left\|\Psi_{1}\right\|\left\|\Psi_{2}\right\|\left\|a_{1}^{*}\left(\bar{h}_{1}\right) \ldots a_{-1}^{*}\left(\bar{h}_{p_{m+n}}\right)\right\| \\
& \cdot\left\|\left(A-A_{l}\right) a_{1}^{*}\left(\bar{h}_{1}\right) \ldots a_{-1}^{*}\left(\bar{h}_{p_{m+n}}\right) \Phi_{0,0}\right\|+\left\|\left(A-A_{l}\right) E_{m, n}\right\|\left\|\Phi_{2}\right\| \\
& \cdot\left\{\sum_{p_{1}+\cdots+p_{m+n}>K}\left|\left\langle\Psi_{1} \mid U_{t} a_{1}^{*}\left(h_{1}\right) \ldots a_{-1}^{*}\left(h_{p_{m+n}}\right) \Phi_{0,0}\right\rangle\right|^{2}\right\}^{\frac{1}{2}} .
\end{aligned}
$$

According to (3.38), the first term in the right-hand side of (3.40) can be made arbitrarily small by choosing $l$ sufficiently large. The second term converges to zero when $t \rightarrow \infty$ since $A_{l} \in \mathfrak{A}_{0}(\tilde{\mathcal{O}})$. Finally, from (3.39), (3.41) and Lemma 3.2 it follows by means of an adequate choice for $K$ that $R(l, t)<\varepsilon$ for all $l$ if $t$ is sufficiently large. Thus, (3.3) holds for any finite linear combination of such elements of $\mathfrak{A}(\tilde{\mathcal{O}})$. Hence, Theorem 3.1 has been established.

\section{Conclusion}

In the preceding section we have established that for any $\Psi \in \mathscr{F}_{ \pm}^{\prime}(\Phi)$ $=\mathscr{F}_{ \pm}(\Phi) \ominus[\Phi]$ there are vectors $\Psi_{ \pm}^{\text {in }}$ out $\in \mathscr{F}$ such that the distribution of spins and moments in the interacting Schrödinger states $e^{-i t H_{\kappa, v}} \Psi_{ \pm}$ 
approaches asymptotically for $t \rightarrow \mp \infty$ the distribution of spins and momenta in the respective free Schrödinger states $e^{-i H_{0} t} \Psi_{ \pm}^{\text {in }}$. If we require that $\Psi_{ \pm}$be normalized with respect to the inner product $\langle\cdot \mid \cdot\rangle_{\text {ren }}$ on $\mathscr{F}_{ \pm}^{\prime}(\Phi)$ and that $\Psi_{ \pm}^{\text {ex }}$ be normalized with respect to the Fock space inner product $\langle\cdot \mid \cdot\rangle$, then

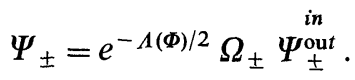

Hence the transition probability amplitude from $e^{-i \mathrm{H}_{\kappa, \nu} t} \Psi_{+}$to $e^{-i H_{\kappa, v} t} \Psi_{-}$is

$$
\left\langle\Psi_{-} \mid \Psi_{+}\right\rangle_{\text {ren }}=\left\langle\Omega_{-}(\Phi) \Psi_{-}^{\text {out }} \mid \Omega_{+}(\Phi) \Psi^{\text {in }}\right\rangle=\left\langle\Psi_{-}^{\text {out }} \mid S(\Phi) \Psi_{+}^{\text {in }}\right\rangle
$$

where we have introduced the scattering operator

$$
S(\Phi)=\Omega_{-}^{*}(\Phi) \Omega_{+}(\Phi) .
$$

We note that the wave-function amplitude renormalization does not affect the $S$-operator. However, $S(\Phi)$ is unitary on $\mathscr{F}$ if and only if $\mathscr{F}_{+}(\Phi) \equiv \mathscr{F}_{-}(\Phi)$ (cf. Ref. [8], Section 2).

We have seen in Ref. [3] that the ground state $\Phi_{\kappa, v}$ of $H_{\kappa, v}$ exists for any $\kappa<\infty$ and integrable $v(\boldsymbol{x})$, but we know neither whether it is unique or whether $H_{\kappa, v}$ has some other eigenvalues besides $E_{\kappa, v}$. Since we have to allow for the possibility that there are more than one linearly independent eigenvector $\Phi$ of $H_{\kappa, v}$, we seem to have to envisage the possibility that there are not only more than one $S$-operators (4.3) which would describe transitions in $\mathscr{F}_{ \pm}(\Phi)$, but also that there should be an $S$-operator describing transitions from $\Psi_{+}$in $\mathscr{F}_{+}\left(\Phi_{1}\right)$ to some $\Psi_{-}$in $\mathscr{F}_{-}\left(\Phi_{2}\right)$, where $\Phi_{1} \perp \Phi_{2}$. However, a meaning could be given to such transition probabilities only if $\Lambda\left(\Phi_{1}\right)=\Lambda\left(\Phi_{2}\right)$. Furthermore, a theory which would possess two or more mutually orthogonal eigenvectors $\Phi_{1}, \Phi_{2}, \ldots$ of $H_{\kappa, v}$ seems to have redundancies at least as far as scattering measurements of spins and momenta are concerned: for any $\Psi \in \mathscr{F}$ we would have two or more interaction states $\Omega_{ \pm}\left(\Phi_{1}\right) \Psi, \Omega_{ \pm}\left(\Phi_{2}\right) \Psi, \ldots$ which would have the same asymptotic distribution of spins and momenta when $t \rightarrow \mp \infty$.

Finally, we should allow for the possibility that there are vectors $\Phi \in \mathscr{F}$ which are annihilated by all $a_{v \pm}(f)$, but which are not eigenvectors of $H_{\kappa, v}$. It is fairly obvious that for such vectors Theorem 3.1 cannot possibly hold in general since already for $E_{0,0}$ we shall not have

$$
\left\langle e^{-i H_{\kappa, v t}} \Phi \mid E_{0,0} e^{-i H_{\kappa, v} t} \Phi\right\rangle=\left\langle e^{-i \eta t} \Phi \mid E_{0,0} e^{-i \eta t} \Phi\right\rangle=e^{-\Lambda(\Phi)},
$$

i.e. we have no non-zero limit when $t \rightarrow \mp \infty$. In fact, if $\Phi$ belongs to the absolutely continuous spectrum of $H_{\kappa, v}$, then the limit of (3.4) as $t \rightarrow \mp \infty$ 
will be zero. However, only if we include in $\subseteq$ [3] all vectors annihilated by $a_{ \pm}(f)$ and choose $\Phi_{1}, \Phi_{2} \ldots$ to be an orthonormal basis in $\subseteq$, can we prove (cf. [5], Theorem 4) that

$$
\mathscr{F} \equiv \bigoplus_{k} \mathscr{F}_{ \pm}\left(\Phi_{k}\right)
$$

On the other hand, if $\Phi$ is an eigenvector of $H_{\kappa, v}$, then the asymptotic behaviour of any $e^{-i H_{\kappa, v} t} \Psi \in \mathscr{F}_{ \pm}(\Phi)$ is that of a state of free noninteracting particles. Hence, in that case $\Omega_{ \pm}(\Phi)$ correspond to channels in which all particles are asymptotically free. It seems plausible to assume that if $\Phi$ is not an eigenvector $H_{\kappa, v}$ then we are dwelling in a channel in which not all particles are asymptotically free, i.e., for which $H_{0}$ is not the channel Hamiltonian.

Acknowledgements. The authors would like to thank Professors D. Masson and P. Rosenthal for discussions and useful remarks.

\section{References}

1. Prugovečki,E.: Nuovo Cimento 4 B, 105 (1971).

2. Manoukian,E. B., Prugovečki,E.: Can. J. Phys. 49, 102 (1971).

3. Prugovečki,E., Manoukian,E.B.: Spin-momentum distribution observables and asymptotic fields for fermion fields with cutoff self-interaction. University of Toronto Preprint (to be published).

4. Kato, Y., Mugibayashi, N.: Prog. Theor. Phys. 30, 103 (1963).

5. Høegh-Krohn, R.: J. Math. Phys. 9, 2075 (1968).

6. - Commun. math. Phys. 18, 109 (1970).

7. Glimm, J., Jaffe, A.: Ann. Math. 91, 362 (1970).

8. Prugovečki, E.: Quantum mechanics in Hilbert space. New York: Academic Press 1971.

9. Glimm, J.: Models for quantum field theory in local quantum theory. In: Jost, R. (Ed.): Local quantum theory. New York: Academic Press 1969.

E. Prugovečki

Department of Mathematics

University of Toronto

Toronto 181, Canada

\author{
E. B. Manoukian \\ Department of Physics \\ University of Toronto \\ Toronto 181, Canada
}

E. B. Manoukian's present address:

Theoretical Physics Institute

University of Alberta

Edmonton, Canada 